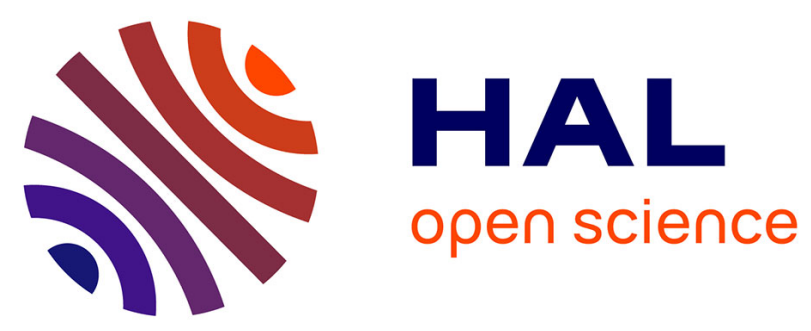

\title{
The real-time distributed control of the Virgo Interferometric Detector of Gravitational Waves
}

F. Acernese, P. Amico, M. Alshourbagy, F. Antonucci, S. Aoudia, P. Astone, S. Avino, D. Babusci, G. Ballardin, F. Barone, et al.

\section{- To cite this version:}

F. Acernese, P. Amico, M. Alshourbagy, F. Antonucci, S. Aoudia, et al.. The real-time distributed control of the Virgo Interferometric Detector of Gravitational Waves. 11th IEEE International Symposium on Distributed Simulation and Real Time Applications, Oct 2007, Chania, Greece. pp.302-310, 10.1109/TNS.2007.912887 . in2p3-00191056

\section{HAL Id: in2p3-00191056 https://hal.in2p3.fr/in2p3-00191056}

Submitted on 23 Nov 2007

HAL is a multi-disciplinary open access archive for the deposit and dissemination of scientific research documents, whether they are published or not. The documents may come from teaching and research institutions in France or abroad, or from public or private research centers.
L'archive ouverte pluridisciplinaire $\mathbf{H A L}$, est destinée au dépôt et à la diffusion de documents scientifiques de niveau recherche, publiés ou non, émanant des établissements d'enseignement et de recherche français ou étrangers, des laboratoires publics ou privés. 


\title{
The real-time distributed control of the Virgo Interferometric Detector of Gravitational Waves
}

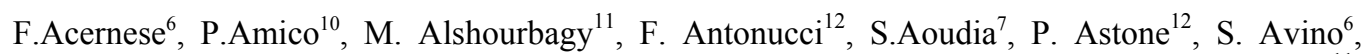 \\ D.Babusci $^{4}$, G.Ballardin ${ }^{2}$, F.Barone ${ }^{6}$, L.Barsotti ${ }^{11}$, M.Barsuglia ${ }^{8}$, F.Beauville ${ }^{1}$, S. Bigotta ${ }^{11}$, \\ M.A.Bizouard ${ }^{8}$, C.Boccara ${ }^{9}$, F.Bondu ${ }^{7}$, L.Bosi ${ }^{10}$, C.Bradaschia ${ }^{11}$, S. Birindelli ${ }^{11}$, S.Braccini ${ }^{11}$, \\ A.Brillet $^{7}$, V.Brisson ${ }^{8}$, D.Buskulic ${ }^{1}$, E.Calloni ${ }^{6}$, E.Campagna ${ }^{3}$, F. Carbognani ${ }^{2}$, F.Cavalier ${ }^{8}$, \\ R.Cavalieri ${ }^{2}$, G.Cella ${ }^{11}$, E.Cesarini ${ }^{3}$, E.Chassande-Mottin ${ }^{7}$, N. Christensen ${ }^{2}$, A.-C.Clapson ${ }^{8}$, \\ F.Cleva ${ }^{7}$, C. Corda $^{11}$, A. Corsi ${ }^{12}$, F.Cottone ${ }^{10}$, J.-P.Coulon ${ }^{7}$, E.Cuoco ${ }^{2}$, A. Dari ${ }^{10}$, V.Dattilo ${ }^{2}$, \\ M.Davier ${ }^{8}$, M. del Prete ${ }^{2}$, R.De Rosa ${ }^{6}$, L.Di Fiore ${ }^{6}$, A.Di Virgilio ${ }^{11}$, B.Dujardin ${ }^{7}$, A.Eleuteri ${ }^{6}$, \\ I.Ferrante $^{11}$, F.Fidecaro ${ }^{11}$, I.Fiori ${ }^{11}$, R.Flaminio ${ }^{1,2}$, J.-D.Fournier ${ }^{7}$, S.Frasca ${ }^{12}$, F.Frasconi ${ }^{11}$, \\ L.Gammaitoni $^{10}$, F. Garufi ${ }^{6}$, E. Genin ${ }^{2}$ A.Gennai ${ }^{11}$, A.Giazotto ${ }^{11}$, G.Giordano ${ }^{4}$, L. Giordano ${ }^{6}$, \\ R. Gouaty ${ }^{1}$, D. Grosjean ${ }^{1}$, G.Guidi ${ }^{3}$, S.Hebri ${ }^{2}$, H.Heitmann ${ }^{7}$, P.Hello ${ }^{8}$, S. Karkar ${ }^{1}$, S.Kreckelbergh ${ }^{8}$, \\ P.La Penna ${ }^{2}$, M. Laval ${ }^{7}$, N. Leroy ${ }^{8}$, N.Letendre ${ }^{1}$, B. Lopez ${ }^{2}$, M. Lorenzini ${ }^{3}$, V.Loriette ${ }^{9}$, \\ G.Losurdo $^{3}$, J.-M.Mackowski ${ }^{5}$, E.Majorana ${ }^{12}$, C.N.Man ${ }^{7}$, M. Mantovani ${ }^{11}$, F. Marchesoni ${ }^{10}$,

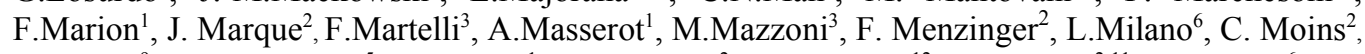 \\ J.Moreau $^{9}$, N.Morgado ${ }^{5}$, B.Mours ${ }^{1}$, F. Nocera ${ }^{2}$, C.Palomba ${ }^{12}$, F.Paoletti ${ }^{2 ; 11}$, S. Pardi ${ }^{6}$ A. \\ Pasqualetti $^{2}$, R.Passaquieti ${ }^{11}$, D.Passuello ${ }^{11}$, F. Piergiovanni ${ }^{3}$, L.Pinard ${ }^{5}$, R.Poggiani ${ }^{11}$, \\ M.Punturo $^{10}$, P.Puppo ${ }^{12}$, K.Qipiani ${ }^{6}$, P.Rapagnani ${ }^{12}$, V.Reita ${ }^{9}$, A.Remillieux ${ }^{5}$, F.Ricci ${ }^{12}$, I.Ricciardi ${ }^{6}$, \\ P. Ruggi ${ }^{2}$, G.Russo ${ }^{6}$, S.Solimeno ${ }^{6}$, A. Spallicci ${ }^{7}$, R.Stanga ${ }^{3}$, M. Tarallo ${ }^{11}$, M. Tonelli ${ }^{11}$, A. \\ Toncelli $^{11}$, E.Tournefier ${ }^{1}$, F.Travasso ${ }^{10}$, C. Tremola ${ }^{11}$, G. Vajente ${ }^{11}$, D.Verkindt ${ }^{1}$, F.Vetrano ${ }^{3}$, \\ A.Viceré ${ }^{3}$, J.-Y.Vinet ${ }^{7}$, H.Vocca $^{10}$, M.Yvert $^{1}$ \\ ${ }^{1}$ Laboratoire d'Annecy-le-Vieux de physique des particules (LAPP), IN2P3/CNRS, Université de Savoie, BP 110, F- \\ 74941, Annecy-le-Vieux, CEDEX, France \\ ${ }^{2}$ European Gravitational Observatory (EGO), Via E. Amaldi, I-56021 Cascina (PI) Italia \\ ${ }^{3}$ INFN - Sezione di Firenze/Urbino, Via G.Sansone 1, I-50019 Sesto Fiorentino and/or Università di Firenze, Largo \\ E.Fermi 2, I - 50125 Firenze, and/or Università di Urbino, Via S.Chiara, 27, I-61029 Urbino, Italia \\ ${ }^{4}$ INFN, Laboratori Nazionali di Frascati, Via E. Fermi, 40, I-00044 Frascati (Roma) - Italia \\ ${ }^{5}$ LMA, 22, Boulevard Niels Bohr, 69622 - Villeurbanne- Lyon Cedex, France \\ ${ }^{6}$ INFN - sezione di Napoli and/or Università di Napoli "Federico II", Complesso Universitario di Monte S. Angelo, Via \\ Cinthia, I-80126 Napoli, Italia and/or Università di Salerno Via Ponte Don Melillo, I-84084 Fisciano (Salerno), Italia. \\ ${ }^{7}$ Departement Artemis - Observatoire Cote d'Azur, BP 42209, 06304 Nice, Cedex 4, France \\ ${ }^{8}$ Laboratoire de l'Accélérateur Linéaire (LAL),IN2P3/CNRS-Université de Paris-Sud, B.P. 34, 91898 Orsay Cedex - France \\ ${ }^{9}$ ESPCI - 10, rue Vauquelin, 75005 Paris - France \\ ${ }^{10}$ INFN Sezione di Perugia and/or Università di Perugia, Via A. Pascoli, I-06123 Perugia - Italia \\ ${ }^{11}$ INFN - Sezione di Pisa and/or Università di Pisa, Via Filippo Buonarroti, 2 I-56127 PISA - Italia \\ ${ }^{12}$ INFN, Sezione di Roma and/or Università "La Sapienza", P.le A. Moro 2, I-00185, Roma
}

\begin{abstract}
The VIRGO experiment for the detection of gravitational waves is a big challenge both for physics and for technology, in particular, to satisfy the stringent requirements on the alignment and position of its suspended optical components to keep the detector at its working point, a very complex distributed and supervised control system has been implemented. The current constraints are about $10^{-10} \mathrm{~m}$ RMS for the longitudinal control ('Locking") and $10^{-9}$ rad RMS for the angular degrees of freedom ("Alignment"). These requirements are satisfied by means of a specially designed hierarchical architecture for the local control system, necessary for managing the hard task of filtering all the environmental noises that limit the sensitivity of the interferometer, supervised by a distributed global control system to maintain the detector fully operational. In this paper we describe the status of the real-time distributed control system of the Virgo interferometric detector of Gravitational waves, its performances and planned improvements.
\end{abstract}

Index Terms-Digital control, real time systems, data acquisition, interferometry, gravitational waves.

\section{INTRODUCTION}

V irgo [1] is a French-Italian experiment aimed at the direct detection of gravitational waves. It is based on a kilometric Michelson interferometer with Fabry-Perot cavities in the arms and light recycling technique in order to improve its sensitivity. 


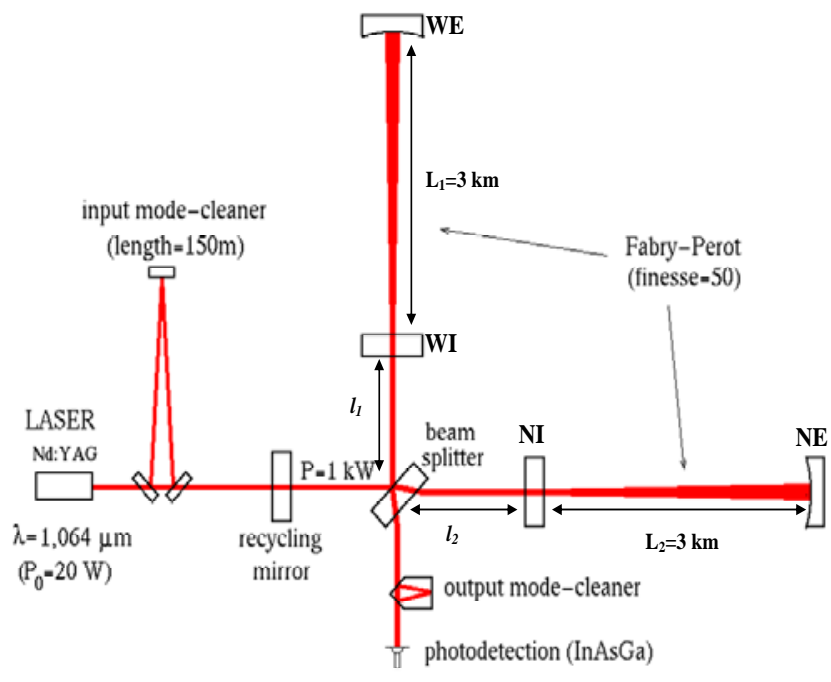

Figure 1: VIRGO interferometer schematics

It can be easily shown that a perfect Michelson interferometer (defined by its arm length $L$ and illuminated by a laser with a power $\mathrm{P}$ and a wavelength $\lambda$ ) reaches its maximum sensitivity when it is tuned on the dark fringe. In the optimal case, the sensitivity to gravitational waves is limited by the photon counting statistics:

$h_{\text {shot }}=1 / 2 L \sqrt{h c \lambda / P \Delta t}$, being $h$ the Planck constant, $c$ the speed of light, $P$ the laser power, $L$ the arm length and $\Delta t$ the observation time. Thus, the longer is the arm, length the better is the sensitivity. In ground based interferometers, the arm length cannot go above the kilometric scale because of the land configuration and the cost of the needed infrastructure. The Virgo interferometer arms are $3 \mathrm{~km}$ long (see Figure 1). In order to increase the optical path within a given physical dimension, two Fabry-Perot cavities are inserted in the arms. When the Fabry-Perot cavity is resonant, the optical length is related to the physical path by: $l_{\text {opt }}=l_{\text {phys }}(2 F / \pi)$, where the

Finesse $F$, is a function of the cavity mirror reflectivities. The Finesse of VIRGO Fabry-Perot cavity is $F_{f p}=50$.

Since in dark fringe condition most of the light is reflected back to the laser, a further mirror, the recycling mirror is added between the beam splitter and the laser, giving rise to another cavity: the recycling cavity that "recycles" the reflected back power, into the interferometer. The Finesse of this new cavity, $F_{R e c}$,is related to the total losses of the interferometer and in the case of VIRGO, is around 50.

\section{CONSTRAINTS}

The foreseen sensitivity imposes that the Fabry-Perot arms and the recycled cavity are resonant, and that the interferometer output is a dark fringe. The longitudinal mirror control to achieve these conditions is called Locking.

The conditions to achieve locking are that the fluctuations on the cavities lengths must be less than $1 / 10$ of the full width to half maximum of the resonance. This leads to the constraints on the Fabry-Perot longitudinal control: $\delta L_{i} \leq \lambda / 4 F_{f p}=5 \cdot 10^{-9} \mathrm{~m}$, and on the Recycling cavity control: $\delta l_{i} \leq \lambda / 4 F_{\text {rec }}=5 \cdot 10^{-9} \mathrm{~m}$.
Even stricter is the condition on the dark fringe; the end mirror displacement variation must be lower than the shot noise fluctuation. This constrains the end mirror position $l_{d}=l_{2}-l_{1}+2 F_{f p} / \pi /\left(L_{2}-L_{1}\right)$ to be within $10^{-10} \mathrm{~m}$.

For the Fabry-Perot cavity to work properly, the cavity mirrors must be aligned; the laser direction jitter couples to the static misalignment of the mirrors and the induced noise can limit the interferometer sensitivity. An analytic calculation leads to the constraints on the Fabry-Perot and recycling mirror angular control: $\delta \theta_{F P \text { end }}<3 \cdot 10^{-9} \mathrm{~m} ; \delta \theta_{F P \text { input }}<2 \cdot 10^{-8} \mathrm{~m}$; $\delta \theta_{\text {rec }}<10^{-7} \mathrm{~m}$.

It is clear that to maintain these conditions on all the mirrors on a kilometric distance is a very complex task, that implies also the mirrors to be as much as possible isolated from all the environmental noises (seismic, tidal, electromagnetic, etc). The goal is achieved by the combination of a mechanical isolation from the ground motions, local mirror controls and a global interferometer control system.

\section{SUSPENSIONS AND LOCAL CONTROLS}

\section{A. Superattenuator and hierarchical control}

To isolate the mirrors from ground motions within the detection band $\left(10-10^{4} \mathrm{~Hz}\right)$, they are suspended to a multi pendular structure: the superattenuator (SA), as shown in Figure 2. The first stage is an inverted pendulum (IP) to the top of which, five mechanical filters are suspended. Each pendular stage, acts as a $2^{\text {nd }}$ order low-pass filter. The full chain is characterized by very low frequency normal modes (all are in the range $0.04-3 \mathrm{~Hz}$ ).

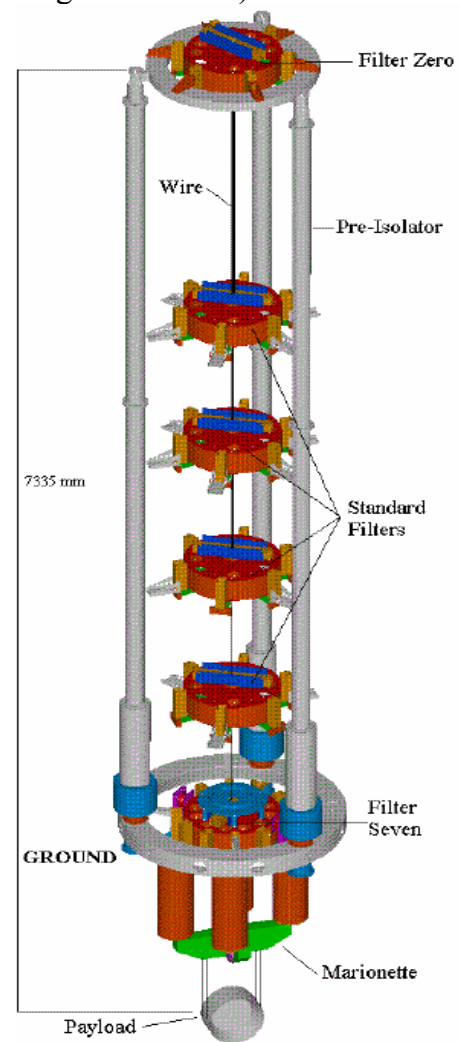

Figure 2: The multipendular suspension of VIRGO mirrors 
In order to get the lock conditions, besides providing a passive filtering of the seismic mechanical vibrations, the SA is designed to allow an active control of the mirror position over a very large dynamic range [2]. To extend the detection band down to $4 \mathrm{~Hz}$, stricter limits on the actuators noise are demanded. In particular, the actuation stages close to the mirror must be free from seismic noise, i.e they must be suspended. Control forces are exerted at three actuation points (see Figure 2):

- The Inverted pendulum top stage is provided by accelerometers and position sensors. Magnetic actuators centre the chain and use the sensor signals for low frequency three dimensional active damping of the resonances of the SA

- The Marionette, by means of magnetic actuators, allows to move the payload with respect to the last stage.

- The reference mass, equipped with coils that act on the mirrors.

The force on the three actuation points is exerted in different frequency bands: below $10^{-2} \mathrm{~Hz}$ on the top stage, between $10^{-2}$ and $5 \mathrm{~Hz}$ on the marionette, and from 5 to $50 \mathrm{~Hz}$ on the reference mass. Moreover, since reaching the work point needs a larger force than maintaining it, a high gain chain is used in the lock acquisition phase while a low gain chain is used in lock conditions.

In the IP control, three linear variable differential transducers (LVDT) and three accelerometers placed on the top-stage of the IP measure respectively the position of the IP relatively to the external frame and the acceleration in the $x, y$ (transverse translations of the chain suspension point in the horizontal plane) and $\theta$ (rotation of the IP top-stage around the vertical direction) degrees of freedom. The obtained error signal matrix is diagonalised in order to reduce the system from a Multiple Input Multiple Output (MIMO) to three Single Input Single Output (SISO). Then, for each d.o.f., the diagonalised accelerometer and LVDT signals are blended using a low pass filter and and high pass filter in order to minimise the reinjection of seismic noise and build up the signal sent to three coil-magnet actuators which correct the $x, y$ and $\theta$ fluctuations. The diagonalization and filtering operation are made on a custom digital signal processing unit (DSP) [3] based on Motorola DSP96002 capable to implement Infinite Impulse Response (IIR) filters with 8 poles and 8 zeros in about 1 microsecond. In addition to IIR filters implementation, the DSP implements signal generators, matrix multiplication, switches, comparators with hysteresis and many others. The sustained computational power available is about 40 Mega Floating Point Operations per Second (MFPLOPS)

\section{B. Local Control}

Proper detector operation needs a pre-alignment and a mirror angular control. This is realised with the local control [4] consisting of a CCD camera and a 2 laser diode injecting light onto the mirror inside each tower vacuum tank. Both the camera and the laser are on the ground outside the tower: the laser is injected through an optical fibre and the camera monitors the light spot through a window. Six error signals, $x$, $y, z, \theta_{x}, \theta_{y}, \theta_{z}$ are referred to the mechanical ground of the CCD-camera and reconstructed. A dedicated read-out board was developed in order to use this CCD-camera for real-time applications in the standard VME crates of Virgo. The CCDchip is a scientific grade square $512 \times 512$ pixel matrix with $7.7 \times 7.7 \mathrm{~mm}^{2}$ surface. The pixel width is $15 \mu \mathrm{m}$. The pixel, clock is set at $20 \mathrm{MHz}$ and the system provides a digital signal through an 8-bit ADC. The image is gathered and converted in $16 \mathrm{~ms}$. Thus, the maximum speed is 60 frames/s. The frame grabbing, triggered by an external timing control, is a key feature of this system integrated in the real-time local control standard crate of Virgo. The accuracy of the CCD position sensing is roughly $10 \mathrm{~nm}$ RMS, on the surface of the CCD chip, with a spot diameter of $30-300 \mu \mathrm{m}$. The readout system described above was designed to accomplish two main tasks:

(A) the control of large angular offsets or oscillations in the pre-alignment phase (coarse-mode);

(B) control during the locking and operation phases (fine-mode).

The pre-alignment phase is obtained by monitoring four ceramic markers illuminated with a halogen lamp. Differential signals are derived from these measurements at $50 \mathrm{~Hz}$ and passed to a DSP until the control accuracy provides $100 \mu \mathrm{rad}$ RMS and $100 \mu \mathrm{m}$ rms. Then, the fine error-signal computation of $\theta_{\mathrm{x}}$ and $\theta_{\mathrm{y}}$, obtained by a linear combination of the variations along the two coordinates read by two Position Sensing Devices (PSD) looking at the reflected laser beam, automatically replaces the coarse computation and the halogen illuminator is switched off. If during the operation the control is switched back from the fine to the coarse-mode, the calibration and the offset of coars signals are automatically updated by matching the last (more accurate) values provided by the fine measurement.

Figure 3 is a picture of the local control crate, while in Figure 4 the main elements of the local control chain are shown. The VME crate is divided in two sections, each equipped with a dedicated CPU board (coded as A and B). The main process running in the CPU-A is the DSP-server, a software program designed to allow real-time communication between two digital signal processing boards (DSP-A and B) and a software program that controls a set of ADC and DAC boards. Both DSP units are also programmed to send data to the data

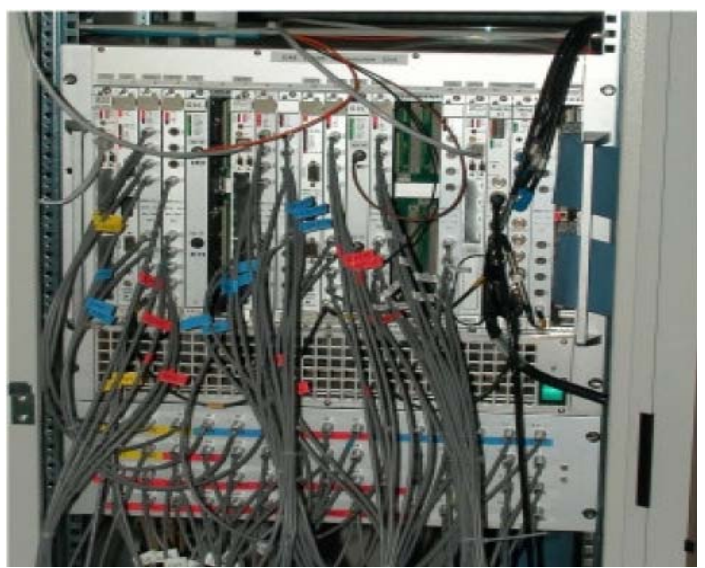

Figure 3: An image of the local control 


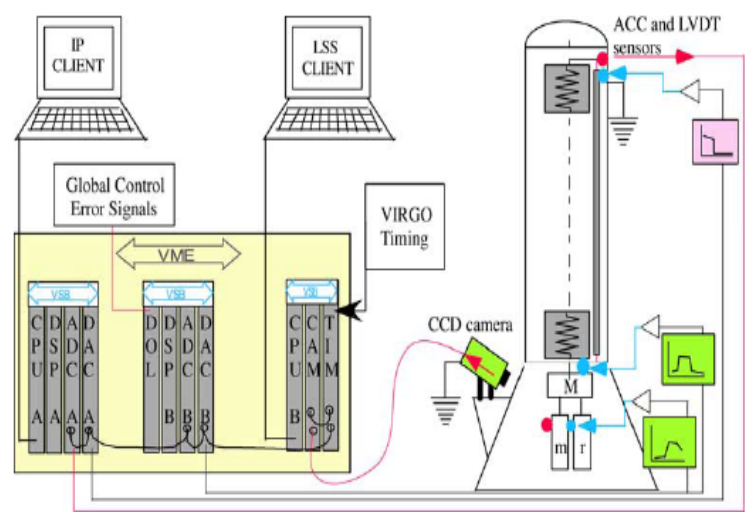

Figure 4: A schematic view of the local control chain. Read-out and actuation points are shown.

acquisition system (DAQ) running elsewhere and to receive signals from the Global control system through digital optical link boards (DOL).

\section{THE Global CONTROL}

The global control [5] is the system in charge of collecting data from the photodiodes implied in locking and alignment, elaborate them and send the correction signals to keep the interferometer at its working point. The Locking loop runs at $10 \mathrm{kHz}$ while the Alignment one is synchronized at $500 \mathrm{~Hz}$. A higher bandwidth is needed for Locking mainly for the lock acquisition phase where feedbacks with high unity-gain frequency are needed. All data are also sent to the Data Acquisition (DAQ) at the acquisition frequency of $20 \mathrm{kHz}$ to be stored in the Raw Data frames.

\section{1) Locking}

The goal of a lock acquisition procedure is to bring the ITF on its working point, by controlling its independent longitudinal lengths.

Four lengths must be controlled (with reference to Figure 5):

1. $\mathrm{MICH}=1_{2}-1_{1}$

2. $\mathrm{PRCL}=1_{\mathrm{rec}}+\left(1_{2}+1_{1}\right) / 2$

3. $\mathrm{CARM}=\mathrm{L}_{2}+\mathrm{L}_{1}$

4. $\mathrm{DARM}=\mathrm{L}_{2}-\mathrm{L}_{1}$

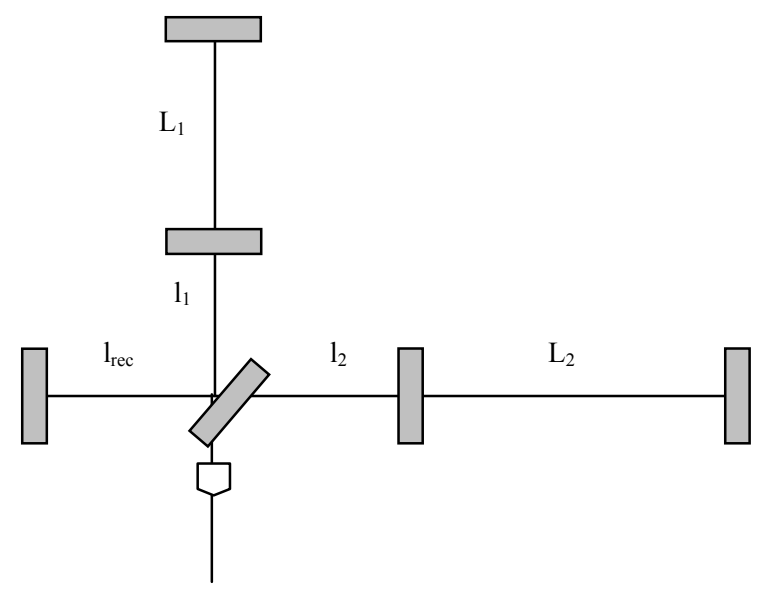

Figure 5: Locking procedure lengths to be controlled
For the ITF locking a novel strategy, called Variable Finesse Locking [6] has been adopted. The basic idea is that the ITF is locked on the half (grey) fringe and then brought sequentially to the dark fringe through several steps. During those steps the control scheme is changed.

The lock acquisition procedure embeds this strategy and consists in two main sequences:

1. Pre-alignment Sequence

2. Locking Sequence

All this is implemented by an Automatic Locking Procedure (ALP) composed of three macros that align the direct beams inside the arms, lock independently the North and West cavities and execute a coarse Power Recycling mirror alignment with respect to the arm cavities mirrors.

Once acquired, the locking is maintained by the Global Control Locking loop.

One of the major Locking loop constraints is to be perfectly synchronised at $10 \mathrm{kHz}$. Any jitter or delay make the system unstable, so, every $100 \mu \mathrm{s}$, the Global Control performs the same series of tasks. First of all, the Global Control checks the time stamp (given by the Virgo Timing system) of the data in order to insure the synchronization and that the photodiode value is inside a defined range in order to avoid overflow problems. Then the lengths are estimated via an optical matrix and translated into individual mirror displacements. This splitting between suspensions can be done using a static driving matrix. However, the driving part can include a splitting in different frequency bands in order to use the natural mechanical gain of the suspensions at some frequencies or to avoid frequency regions where a given suspension has a notch in its transferr function. Once computed, all corrections and triggers are sent using optical links to the suspensions and the Input Bench.

\section{a) The error signals for the Locking}

The Pound-Drever method [7] has been developed to control the length of a Fabry-Perot cavity illuminated by a laser. The technique consists in phase modulating the incoming beam at a frequency $\Omega$, chosen in order to have the sidebands antiresonant when the carrier is resonant. Then, the error signals are obtained by looking at the demodulated components of the reflected and transmitted beams. A length modification induces a beating between the carrier and the side-bands which is the source of the error signal. Near the resonance, the error signal depends linearly on the displacement toward the resonance and the slope is fully determined by the optical characteristics of the cavity.

\section{2) Alignment}

The Alignment feedback loop runs at $500 \mathrm{~Hz}$. After accessing the 80 quadrant photodiode signals, the Global Control reconstructs the angles using a chi-square minimization (with a linear model) or simple matrix inversion. Then, these values are sent to the suspensions and the input bench. As for the Locking case, this splitting can also be done in the frequency domain.

Since there is only one channel from Global control for each suspension for both the longitudinal and angular control, the 
alignment corrections are sent to suspensions at the locking frequency $(10 \mathrm{kHz})$ although they are refreshed at the alignment frequency $(500 \mathrm{~Hz})$.

\section{a) The angular error signals}

When a perfect Gaussian beam (pure TEM00) impinges on a tilted mirror, some high order modes are generated. If the tilt angle is much less than the beam divergence, in practice, only the first mode (TEM 10 or TEM01) is generated. The error signal used for the control is proportional to the product TEM $00 \times$ TEM 10 which gives no signal on a standard photodiode. So, it is necessary to use a quadrant photodiode to extract the spatial information, using the differences between horizontal and vertical quadrants.

For the Virgo Alignment a differential wave front sensing technique (the Anderson-Giordano technique [8]) is adopted. For each beam, two quadrant photodiodes (QPD) sense the wave front change at the laser waist position and at the far end. The modulation frequencies for Alignment and Locking are identical. Once the interferometer is longitudinally locked, the angular error signals are related to the various misalignments through a matrix like in the longitudinal case.

In Figure 6, the Global control read-out schematic is shown, while in Figure 7, the main actors in Global Control chain and their interconnection are outlined: Signals are sent to the input bench control for the second stage of laser frequency stabilization (SSFS)

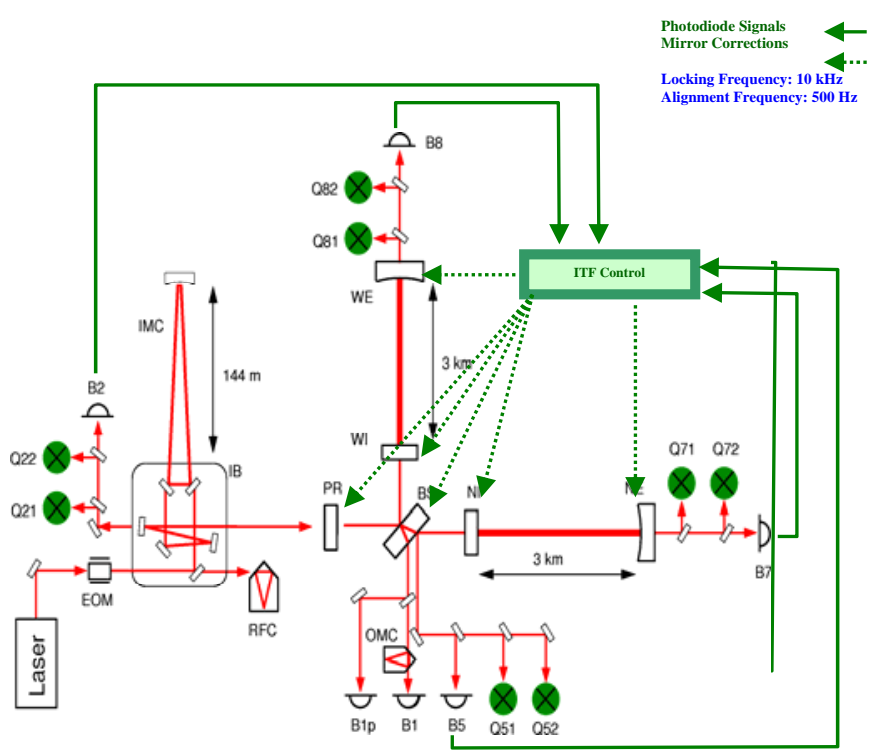

Figure 6: The Global Control locking and alignment readout schematics. Q's are the quadrant photodiodes, B's the beams read-out.

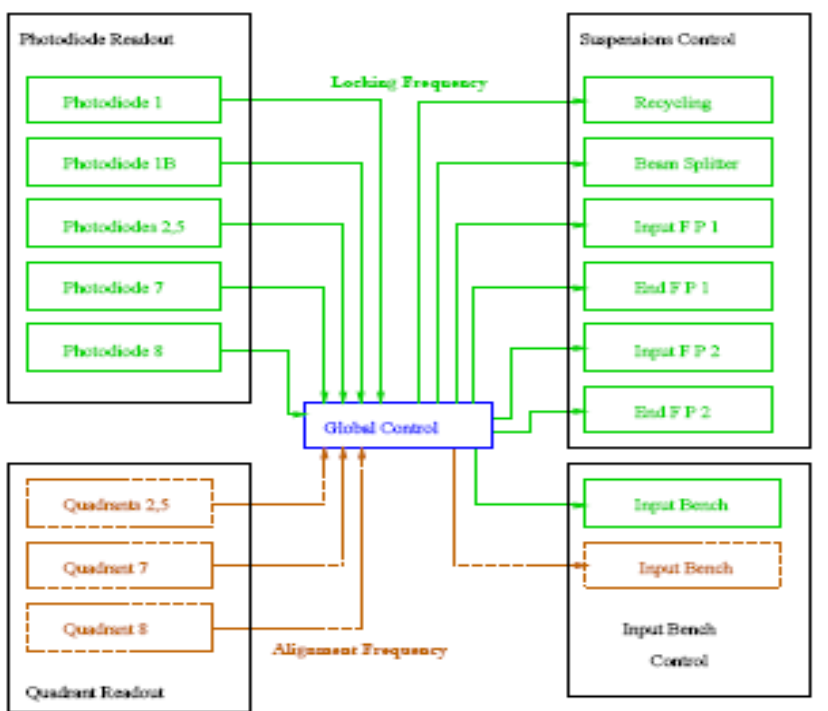

Figure 7: The actors of the Virgo control. The control chain is composed by the photodiode readouts, the Global control, the Suspensions controls and the Input Bench control.

3) The Global Control hardware architecture

The Global Control hardware consists of three components: a crate housing the hardware dedicated to the Locking feedback loops, a second crate for the Alignment control loop and a workstation monitoring the system.

The philosophy underlying the hardware choices is to split the functions into mostly independent $\mathrm{CPUs}$ and processes to minimize the interference on the fastest feedback loop and to build a flexible architecture which can easily evolve.

The technical constraints for the global control are given by the large number of signals it has to read from a large number of boards ( 25 words on 8 boards for locking and 83 signals on 3 boards for alignment), the correction signals to be written (39 for locking and 17 for alignment), the data to be transferred between systems, and the control loop timing.

The standard bus of choice for VIRGO is VME [9] with VSB for high speed data transfer. VSB is hosted on the same crates of VME via piggy-back circuits. The VME CPU used by global control is the RIO8062 [10] by CES [11], based on Motorola PowerPC604 CPU and a custom designed VME to PCI bridge. The operating system is LynxOS by LinuxWorks [12], a real-time Unix-like POSIX conformant operating system for embedded applications, in version 2.5.1.

The various subsystems of VIRGO exchange data via optical fibres, connected through a custom Digital Optical Link (DOL) board [13]. It is a VME/VSB module capable of sending small amount of data (some kByte) in a point-to-point connection over distances up to $3.2 \mathrm{~km}$ with virtually no latency. All the VIRGO subsystems are synchronised by means of a central clock derived from a GPS receiver and custom timing boards. The central clock is delivered through optical fibres to the different buildings and the timing boards convert it into TTL signals and VME bus interrupts for local use. 


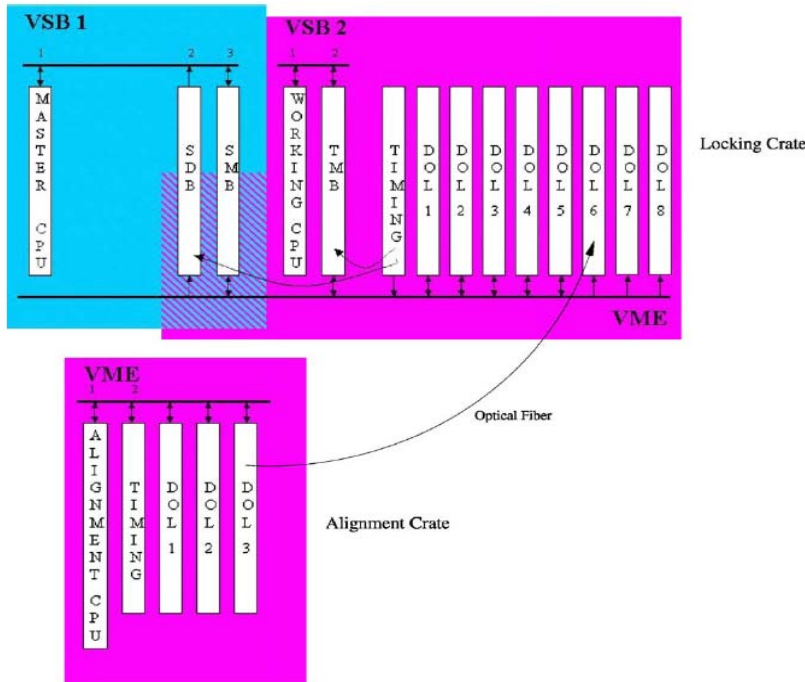

Figure 8: A schematic view of RIOT Global Control crates

To reach the desired performance it has been necessary to design a Transparent Memory Board (TMB) that allows the CPUs to read/write at a single address and acts as a transparent

bridge between the VSB and the VME for the distribution of data, triggered by the timing signal. Moreover a custom VSB LynxOS driver has been written, because the CES driver was poorly performing for the needs of Global Control.

With the described hardware, the Global Control can match the $10 \mathrm{kHz}$ constraint, but the CPU time remaining for other operations such as monitoring is not enough. Thus a SPY Data Board (SDB) has been built, to sniff the data read/written by the TMBs on the VME and present them on the VSB in such a way that another CPU, dedicated to locking monitoring, can access data without disturbing the locking loop

In Figure 9, a schematic view of the Real Time Global control (RIOT) is shown. The SMB is a shared memory (dual port VME/VSB) that allows the "working" CPU to access commands and write status from/to the master.

4) The Global Control Software Architecture

All the VIRGO on-line applications are built following the client-server scheme.
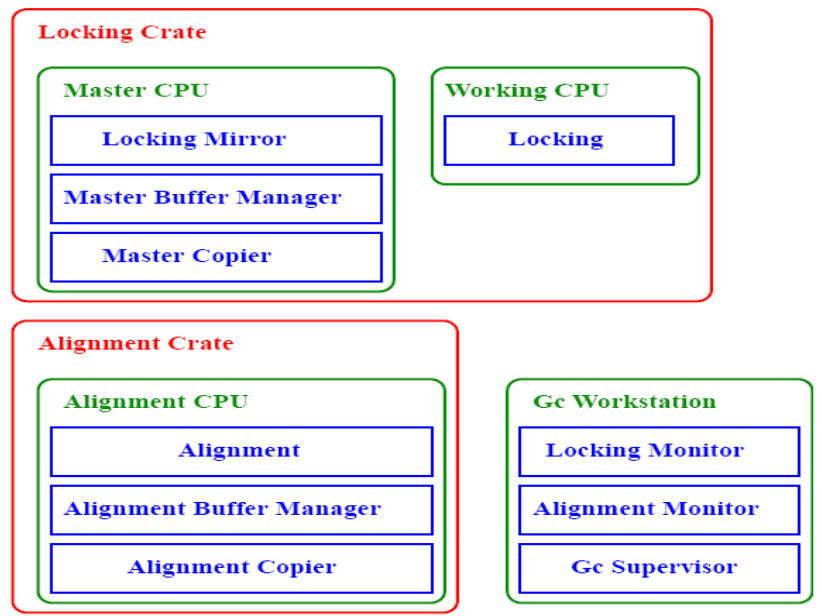

Figure 9: A schematic view of Global Control software architecture

The Global Control is composed by various servers, as shown in Figure 9: two main processes in charge of handling the Alignment and the Locking fast loops, a supervisor process and monitoring processes.

The GC Supervisor handles the whole Global Control in a centralized way and its graphical user interface gives information about the servers' state and allows steering the final state machines associated to each server. All other processes are dedicated to monitoring, and share the data with one another on the same CPU through a shared memory, in order to minimize the interference between the monitoring and control processes. To manage accesses to he shared memory with a producer-consumer model, a buffer manager has been developed, capable of handling several producers and consumers at the same time.

Once the Copier consumer acquires the data from the buffer manager, it sends them to the monitoring processes via TCP/IP, with the help of the Cm package [14]. This package has been built with the aim to permit the communication between the processes hiding to the user the complexity of the TCP/IP programming.

The Locking server has to handle the data exchange between locking crates with the DOLs and apply the control algorithms. The locking algorithm is logically split in four parts:

1. Sensing: computes the lengths from the photodiodes signals. Although this is rather simple near the working point, during the lock acquisition procedure it is not simply based on an optical matrix but requires a powerful algorithm that requires that the optical matrices must be estimated at each step of the procedure and inverted at the Locking frequency.

2. Filtering: applies the time domain correction filters to the lengths computed by sensing

3. Boost: Applies several integrator filters to suppress the DC component of the error signal. It is used only after the lock acquisition phase is completed.

4. Driving: The various filtered lengths are distributed to the mirrors according to the driving matrix, supplied by the user.

All the free parameters of the algorithm (optical gain, filter parameters, etc.) are stored in the VIRGO on-line database [15], as well as the history of parameter changes, so that any change of the behaviour of the algorithms can be applied and reverted without modifying the code.

The alignment server is built with the same criteria, but consists of only two parts: sensing and filtering.

\section{FUTURE DEVELOPMENTS}

The suspension control, in use since 1998, is now approaching its limits in terms of both computational power and converter dynamical range. A new control system [3], now under development, foresees multi-DSP computing units, faster and higher resolution $\mathrm{ADCs}$ and DACs, and highly dynamic power drives for coil magnet pairs. Operations up to 100 
kSamples per second in high resolution or 2 MSamples per second at lower resolution will be available with the new system.

The new DSP board shall be constituted by a carrier VME board hosting up to three PMC mezzanine board on its front size, while the back side can host two PC104Plus CPU modules. It is based on the Analog Devices SHARC ADSP$21160 \mathrm{~N}$ at $100 \mathrm{MHz}$ clock speed, Each mezzanine contains six DSP devoted to different tasks, and their mutual connection topology reflects it.

In parallel to the new DSP architecture, a new coil driver scheme has already been tested on the suspension [16] and is currently in use. This scheme is based on the use of two different chains for lock acquisition and linear lock phases. In lock acquisition a higher current is needed and some more noise is tolerated; this is obtained with DAC1 and a transconductance amplifier. After the lock is acquired, DAC1 is switched off and DAC2 is enabled to keep the lock. A further noise reduction is achieved in this second chain by inserting a digital high-pass filter (emphasis) before converting the signal into analog domain for then low-pass filtering in the coil driver. De-emphasis filter can be inserted in front of coil driver.

To improve the immunity to electromagnetic noise, the ADC and DAC boards, presently in the VIRGO data acquisition room and connected to the coil drivers with $30 \mathrm{~m}$ of cable, will be hosted on-board the new coil driver, near the actuation point.

\section{CONCLUSIONS}

The high demanding constraints of the VIRGO experiment, gave rise to the necessity of a very complex supervised and distributed control system. To realize the VIRGO control system, several digital VME/VSB boards and a DSP have been designed and realized at the time the control has started to work. With the available technology VIRGO is able to approach the desired sensitivity, and plans to reach it in the next year.

At present, the technology allows improvements that are already in an advanced stage.

\section{REFERENCES}

[1] G. Losurdo et al., Rev. Sci Instrum. 72 (9), 3653-3661, 2001

[2] F. Acernese et al. Astroparticle Physics 20 (2004) 629-640

[3] A. Gennai: Virgo Note VIR-SPE-PIS-4900-120, 2004

[4] F. Acernese et al. Astroparticle Physics 20 (2004) 617-628

[5] N. Arnaud et al. Nucl. Instr. and Meth. A 550 (2005) 467-489

[6] A. Acernese, et al., Class. And Quantum Grav, 23 (2006) S85-S89

[7] R.W.P. Drever et al., Appl. Phys. B31 (1983) 97

[8] D. Babusci, et al. Phys.Lett. A. 226 (1997) 31-40.

[9] W. D. Peterson, VMEbus Handbook, fourth ed., VITA, 1997

[10] RIO2 8062: PowerPC based RISC I/O Boards, DOC 8062 UM CES, 1998

[11] CES - Creative Electronic Systems S.A. 38 avenue Eugène-Lance PO Box 584 CH-1212 Grand-Lancy 1 SWITZERLAND

[12] LynuxWorks 855 Embedded Way, San Jose CA 95138-1018 U.S.A.

[13] D. Boget, F. Bellachia, B. Mours, D. Verkindt, Virgo Note VIR-SPELAP-5200-105.

[14] C. Arnault, P. Massart:Virgo Note VIR-MAN-LAL-5100-112, 1998

[15] C. Arnault: Virgo Note VIR-MAN-LAL-5600-111

[16] A. Gennai: Virgo Note VIR-SPR-PIS-4900-121, 2004 